\title{
Clinical Utility of Tumor Markers
}

\author{
Taro Mizuno1, Takayuki Goto², Kota Shimojo ${ }^{3}$, Naoki Watanabe ${ }^{4}$, Takuji Tanaka4,5* \\ ${ }^{1}$ Department of Medical Oncology, Nagoya Medical Center, Nagoya, Japan \\ ${ }^{2}$ Department of Hematology, Gifu Municipal Hospital, Gifu City, Japan \\ ${ }^{3}$ Department of Internal Medicine, Gifu Municipal Hospital, Gifu City, Japan \\ ${ }^{4}$ Department of Diagnostic Pathology \& Research Center of Diagnostic Pathology, Gifu Municipal Hospital, Gifu City, Japan \\ ${ }^{5}$ Center of Genomic Medicine, Gifu Municipal Hospital, Gifu City, Japan \\ Email: *takutt@gmhosp.gifu.gifu.jp
}

How to cite this paper: Mizuno, T., Goto, T., Shimojo, K. and Watanabe, N. (2021) Clinical Utility of Tumor Markers. Open Journal of Pathology, 11, 38-57. https://doi.org/10.4236/ojpathology.2021.1 $\underline{12005}$

Received: March 24, 2021

Accepted: April 27, 2021

Published: April 30, 2021

Copyright $\odot 2021$ by author(s) and Scientific Research Publishing Inc. This work is licensed under the Creative Commons Attribution International License (CC BY 4.0).

http://creativecommons.org/licenses/by/4.0/

\begin{abstract}
Tumor markers comprise a wide spectrum of biomacromolecules excessively synthesized by a variety of neoplastic cells. These markers can be endogenous products of highly active metabolites from malignant neoplastic cells or the products of newly activated genes. Ideally, tumor markers should be highly sensitive, specific, and reliable with a high prognostic value and organ specificity. In addition, they should reflect the tumor stage. However, no tumor markers identified thus far have all of these characteristics. Nevertheless, most tumor markers show excellent clinical relevance for monitoring the efficacy of a variety of therapies. We herein review how to use the recommended tumor markers to diagnose malignancies, such as gastrointestinal carcinoma, liver cancer, bile duct/pancreatic cancer, lung cancer, breast cancer, gynecologic cancer, and urologic cancer.
\end{abstract}

\section{Keywords}

Tumor Marker, Neoplastic Cells, Tumor Stage, Prognosis, Monitor, Therapeutic Effects

\section{Introduction}

Because of the low diagnostic sensitivity and specificity of tumor markers, they cannot detect the early presence of cancers. As such, under the Japanese health insurance system, tumor marker assessments are instructed to be performed only in patients in whom a malignant tumor is strongly suspected based on medical examinations and other imaging study results. Examining pairs of tumor markers in a "combination assay" improves the diagnostic sensitivity, but the diagnostic specificity decreases. However, tumor markers are extremely useful for 1) 
determining the staging of cancer; 2) determining the histologic type of cancer; 3 ) determining the surgery and chemotherapy; and 4) the early detection of recurrent cancer when they are positive; therefore, these markers are an essential tool in the clinical detection of cancer.

\section{Evaluation of Tumor Markers Based on Clinical Epidemiology}

An appropriate sensitivity and specificity are necessary for the evaluation of tumor markers, but such factors alone are useless in the clinical setting. In daily practice, physicians simply want to know the probability of cancer being present when a tumor marker test is positive or the positive predictive value (PPV).

The PPV is important for evaluating the diagnostic efficiency of a tumor marker. The PPV (probability after testing) is determined by the prevalence (probability before testing) and efficacy (sensitivity and specificity) of the testing. Of note, when test results are positive but cancer is not found, this is known as a false positive and is expressed as the False Alarm Rate-1-PPV. We can calculate the colorectal cancer PPV of 1000 subjects in a public medical office and cancer-specific hospital using the CEA which has a diagnostic sensitivity of 50\% - $0 \%$ and diagnostic specificity of $80 \%$ [1]. The prevalence of colorectal cancer is estimated to be $0.5 \%-0.8 \%$ among people without symptoms who undergo total colonoscopy during a multiphasic health screening test. For the convenience of calculation, we considered the prevalence at the general medical office to be $1 \%$ and that at the cancer-specific hospital to be $10 \%$ and then calculated the PPV. When using CEA at a general medical office, which has a low prevalence, the PPV is only approximately $4 \%$, indicating that $96 \%$ of patients will be mistakenly warned about their cancer state. Not only is it useless to undergo an examination with a false positive rate that large, being mistakenly notified of a positive result on such a tumor marker test can induce a substantial economical and psychological burden. However, the PPV increases markedly to $31 \%$ in a cancer-specific hospital with a high prevalence, so the validity of such tumor marker testing is acceptable.

\section{Classification of Tumor Markers}

Tumor markers can be divided into first-generation markers (before 1960: BenceJones protein, human chorionic gonadotropin [hCG], etc.), second-generation markers (the 1960s: $\alpha$-fetoprotein [AFP], carcinoembryonic antigen [CEA], and other embryonic proteins), third-generation markers (1970-1980: carbohydrate antigen [CA]19-9 and other carbohydrate antigens discovered as the results of the monoclonal antibody technology), and fourth-generation markers (post-1990s: human epidermal growth factor receptor type 2 [HER2] protein, p53 protein, and other oncogene/anti-oncogene products). Practical classification, by contrast, describes these elements as non-organ-specific and organ-specific markers. The non-organ-specific markers are CEA, tissue polypeptide antigen (TPA), and 
ferritin, being positive for cancers in various organs. Tumor markers are one of the methods for predicting or diagnosing tumors (Figure 1). As listed in Table 1 and Figure 2, organ-specific markers include AFP, prostate-specific antigen (PSA), neuron-specific enolase (NSE), and others. In addition, some tumor markers have carbohydrate chains and can be divided into type- 1 carbohydrate chains (CA19-9 for pancreatic cancer and gastrointestinal cancer; CA50 for pancreatic, biliary tract and liver cancers; Span-1 for pancreatic, biliary tract and liver cell cancers; KMO-1 [2], pancreatic, biliary tract and liver cancers; and pancreatic cancer-associated antigen-2 [Dupan-2] for pancreatic, biliary tract and liver cancers), type-II carbohydrate chains (sialyl Le[x] antigen [SLX] for lung cancer, Anti-sialyl-Lex[x] mAb [CSLEX] for breast cancer), mother nucleus

Development of malignant tumor

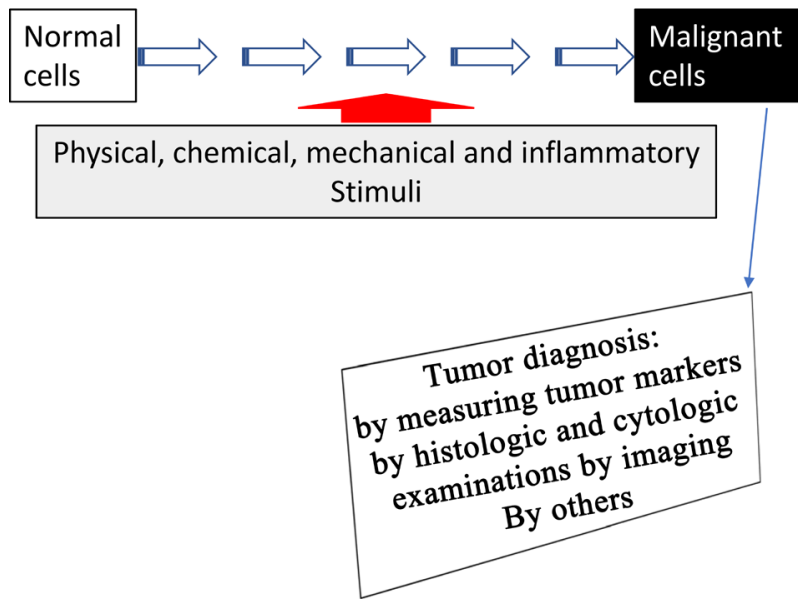

Figure 1. Tumor development and methods of diagnosis.

Table 1. Major tumor markers for cancers in various tissues (non-organ-specific and organ-specific markers).

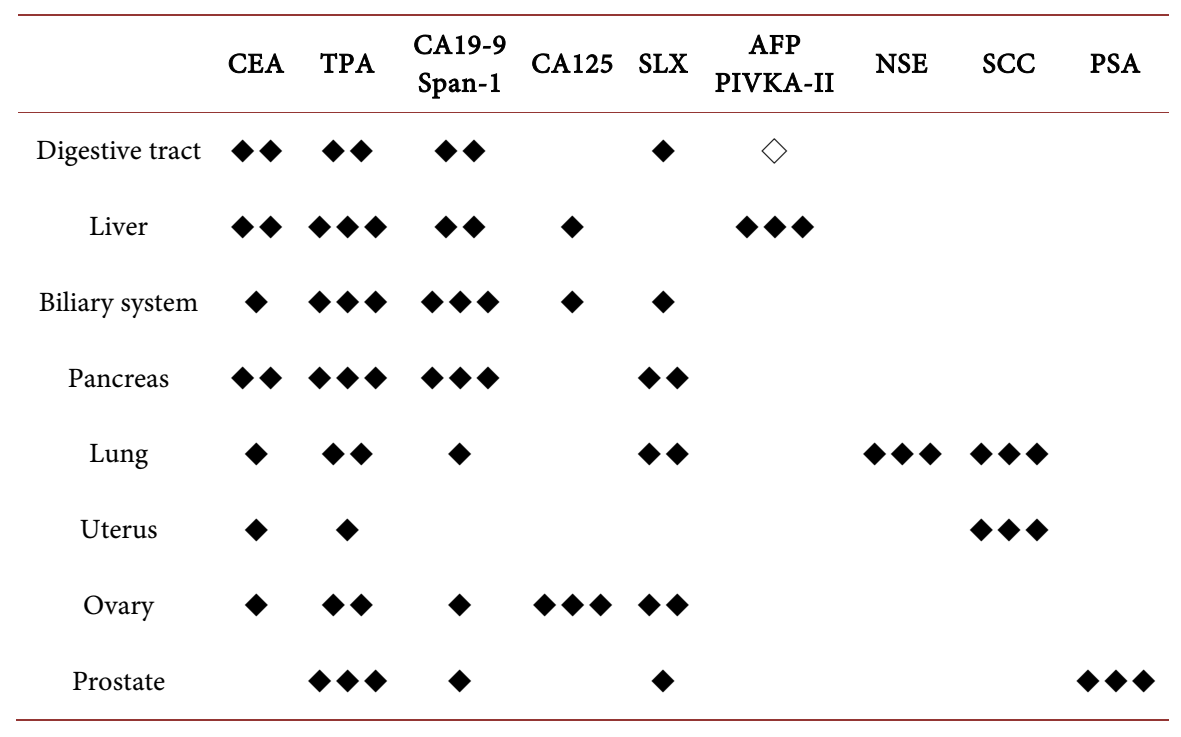

$\diamond \diamond \diamond$ : high positive rate, $\diamond \diamond$ : relatively high positive rate, $\diamond$ : relatively low positivity, but there is supporting diagnostic value and $\diamond$ : AFP-producing gastric cancer. 


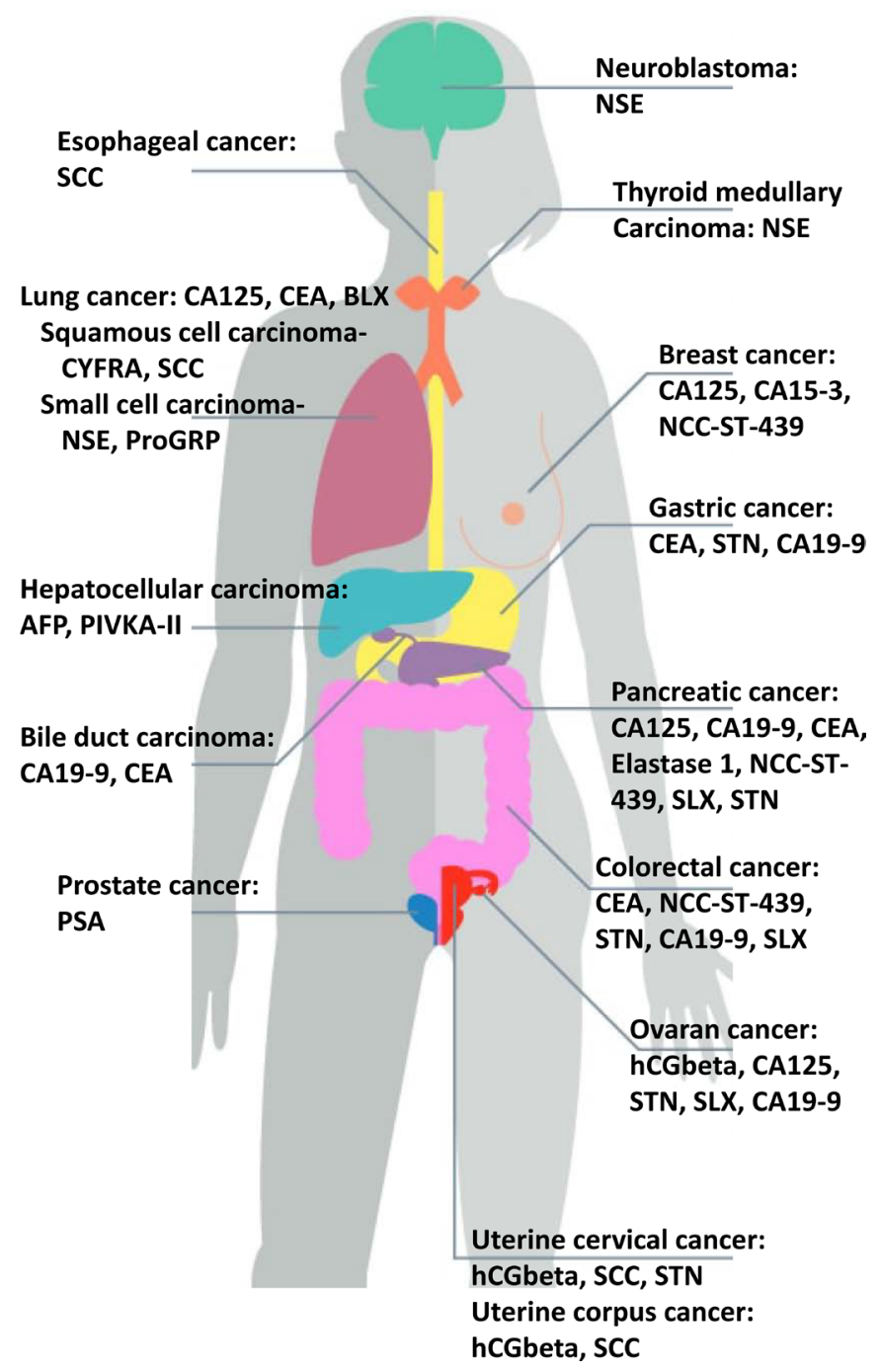

Figure 2. Examples of several tumor markers in tissues.

carbohydrate chains (STN for ovarian cancer; CA72-4 for ovarian and breast cancers; CA546 for ovarian serous cystadenocarcinoma), and core proteins (CA125 for ovarian cancer, CA602 for ovarian serous cystadenocarcinoma; and CA130 for ovarian, uterus, pancreatic and biliary tract cancers) based on the structure of the antigen determinant.

\section{Utility of Tumor Markers According to Organs}

1) Digestive tract carcinoma

Useful markers of the digestive tract are squamous cell carcinoma (SCC) antigen (for esophageal cancer), CEA (for gastric cancer), and CEA (for colorectal cancer). SCC antigen is useful for detecting esophageal cancer but has little significance for its pathological diagnosis. Regarding the utility of tumor markers for gastric cancer, while the significance of their presence for the diagnosis is small, they are useful for the preoperative prediction of the progression stage and postoperative follow-up. CA125 is useful for predicting peritoneal dissemi- 
nation; CEA, CA19-9, and AFP are useful for predicting liver metastasis; and CEA and CA19-9 are useful for predicting lymph node metastasis. Regarding colorectal cancer, CEA is useful for diagnosing its presence, predicting progression, and performing follow-up. CA19-9 is useful for predicting liver metastasis.

- CEA

CEA was first identified in 1965 by Canadian scientists Gold and Freedman in human colon cancer tissue extract [3]. CEA is a glycoprotein of about 200,000 molecular weight related to a cell adhesion factor and belonging to the CEA family. Gold and Freedman initially described it as a carcinoembryonic antigen; however, it was found to be present in not only colorectal cancer tissue but also the gastrointestinal tract, liver, and pancreas of fetuses aged two-six months of age. False positive report is found because it is slightly increased by aging and/or smoking. CEA is positive findings are occasionally reported because CEA levels increase slightly with aging and/or smoking. CEA is positive in $30 \%-70 \%$ of cancers that develop in endodermal tissues, and its positive rate is particularly high in gastrointestinal cancers. As cancer progression, the value of CEA increases. As noted, the CEA positive rate correlates well with the Duke staging of colorectal cancer (Figure 3). CEA as well as CA19-9 are commonly used as tumor markers, although neither demonstrate high diagnostic accuracy [4] [5].

2) Liver cancer

Tumor markers with high specificity for hepatocellular carcinoma (HCC) include AFP and protein induced by vitamin $\mathrm{K}$ absence or antagonist-II (PIVKA-II) [6] [7]. AFP is a representative carcinoembryonic antigen, normally produced in fetal liver and saccus vitellinus. It is not produced after birth. AFP is a glycoprotein comprising $4 \%$ of sugar with a molecular weight of 65,000 . PIVKA-II is abnormal prothrombin produced under vitamin $\mathrm{K}$ deficiency through a prothrombotic precursor.

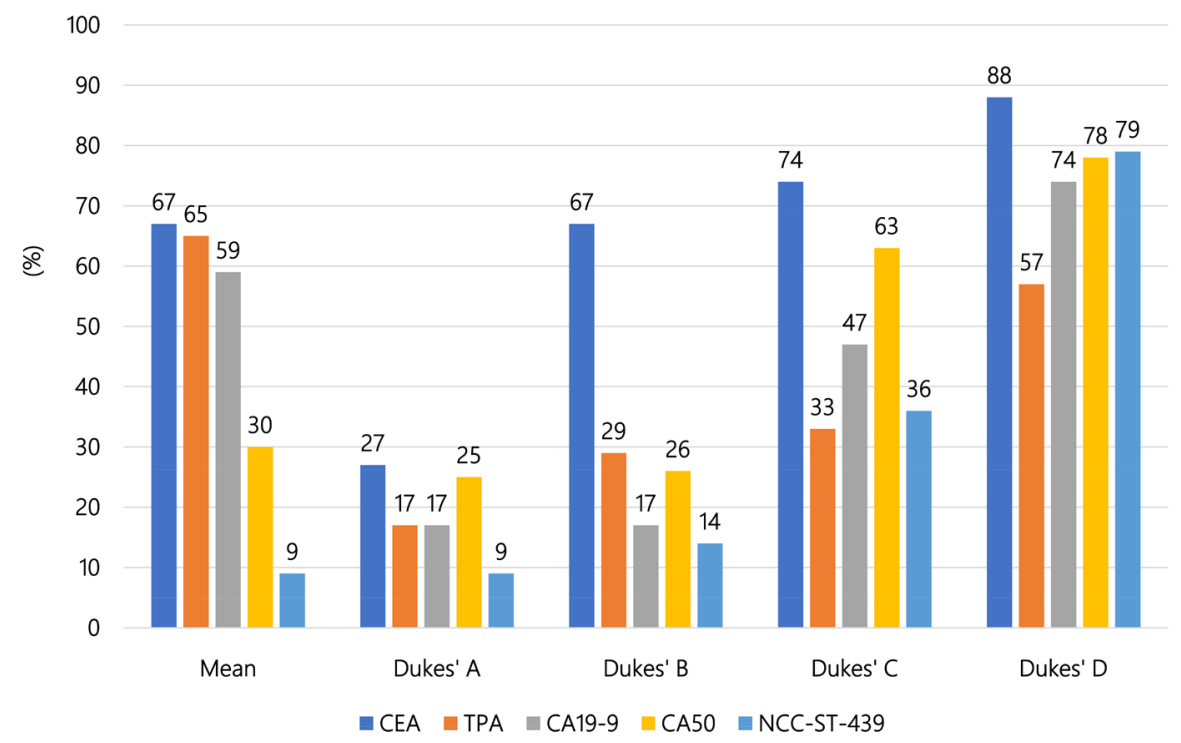

Figure 3. Tumor markers for predicting colorectal cancers. Mean positive rate (\%), Duke staging, and positive rate. 
Because some benign liver diseases are AFP-positive, it is difficult to differentiate liver cancer based solely on the presence of AFP. In the serum of patients with HCC the carbohydrate chain of the AFP is altered, and AFP lectin-responsive fractionation (AFP-L3) increases. No tumor markers have high specificity for primary cholangiocarcinoma, but serum PIVKA-II is increased in such patients. CEA has high sensitivity for metastatic liver cancer.

There have been few studies on the correlation between AFP and PIVKA-II and vascular invasion, tumor differentiation, and size, and conclusions thus far have been controversial [8] [9] [10]. Si et al. [6] reported that AFP and PIVKA-II play a significant role in the diagnosis of HBV-related HCC. Of note, the diagnostic value of AFP and PIVKA-II combined detection or a single assay of PIVKA-II is higher than that of a separate assay for AFP. Furthermore, the concentrations of AFP and PIVKA-II have important clinical value for judging the tumor size, tumor cell differentiation, and vascular invasion.

Huang et al. [11] showed that PIVKA-II combined with AFP had better diagnostic utility than AFP alone for the HCC diagnosis. However, Tarao et al. [7] confirmed the limited ability to detect HCC in patients with very small single HCC nodules ( $\leq 2 \mathrm{~cm}$; Barcelona clinic liver cancer stage 0) [12] [13], even with the combination of both AFP and PIVKA-II.

\section{- AFP}

Serum AFP is positive in $90 \%$ of patients with HCC. The AFP-positive rate of patients with stage IV HCC is $50 \%$, but it cannot be used for the early diagnosis of HCC. During differentiation from benign liver diseases, AFP-positive cases are found among patients with hepatitis and liver cirrhosis. Among patients with chronic hepatitis and liver cirrhosis, $20 \%$ - 40\% are positive for AFP, with 5\% $10 \%$ of patients having serum AFP $>100 \mathrm{ng} / \mathrm{ml}$, although the frequency of patients with $400 \mathrm{ng} / \mathrm{ml}$ is low. The serum value of AFP is not correlated with the size of the HCC; indeed, well-differentiated and undifferentiated HCCs show a low production of AFP, while moderately differentiated trabecular-type HCCs have high values.

Digestive system cancers, testicular cancers, and ovarian cancers sometimes also show high levels of AFP. There have been many case reports on AFP-producing gastric cancers, with cases histologically proving to be hepatoid adenocarcinoma with a poor prognosis. AFP is used for the follow-up of liver cirrhosis, which is a preceding state of HCC.

AFP is a glycoprotein that is often associated with HCC. However, AFP levels also increase during pregnancy, and some benign diseases, such as severe hepatitis and cirrhosis, show increased levels as well. Furthermore, AFP is not significantly increased in about $35 \%$ - $40 \%$ of HCC patients, especially in cases with small HCC [14] [15].

- PIVKA-II

The positive rate of PIVKA-II in HCC is 50\%, but the diagnostic efficiency improves by combination measurement with AFP, as there is no correlation be- 
tween PIVKA-II and AFP. The administration of warfarin and antibiotics increases the serum value of PIVKA-II. AFP is sensitive, but its specificity is low, while PIVKA-II has low sensitivity, but its specificity is high, as shown in Figure 4.

PIVKA-II is an abnormal form of prothrombin, which has been used as a good diagnostic biomarker for HCC [16] [17] [18]. There is now considerable evidence that PIVKAII is an independent prognostic factor after liver surgery, such as hepatic resection or liver transplantation [19]. In addition, PIVKA-II is influenced by many non-tumor factors, such as coagulation dysfunction and liver cirrhosis [16].

- AFP-L3 ([20])

AFP from HCC displays a different affinity for the lectin lens culinaris agglutinin (LCA) from that of chronic hepatitis/liver cirrhosis. Based on the binding capability to LCA, total AFP can be separated into three different glycoforms, AFP-L1, AFP-L2, and AFP-L3. AFP-L1 is the non-LCA-bound fraction, constituting the major glycoform of AFP in serum of chronic hepatitis and liver cirrhosis. AFP-L3 is the LCA-bound fraction of AFP; malignant liver cells reportedly produce AFP-L3, even when HCC is in its early stage, and especially when the tumor mass is supplied by the hepatic artery. Clinical research has suggested that AFP-L3 is a highly specific marker for HCC. AFP-L3 can be detected in the serum of approximately $35 \%$ of patients with small HCC $(<2 \mathrm{~cm})$. AFP-L3-positive HCC has the potential for rapid growth and early metastasis. AFP-L3 can detect HCC earlier 9 to 12 months than imaging detection. The combined sensitivity of AFP-L3 for HCC is $56 \%$, with a specificity of $>95 \%$.

3) Pancreas and bile duct carcinoma

CA19-9, also called sialyl Lewis antigen A, is widely used for the diagnosis and treatment monitoring of pancreatic ductal carcinoma, which has the highest incidence among pancreatic cancers. CEA, SLX, Nation Cancer Center-Stomach-439 (NCC-ST-439), Sialyl Thomsen-nouveau (STN), pancreatic cancer-associated

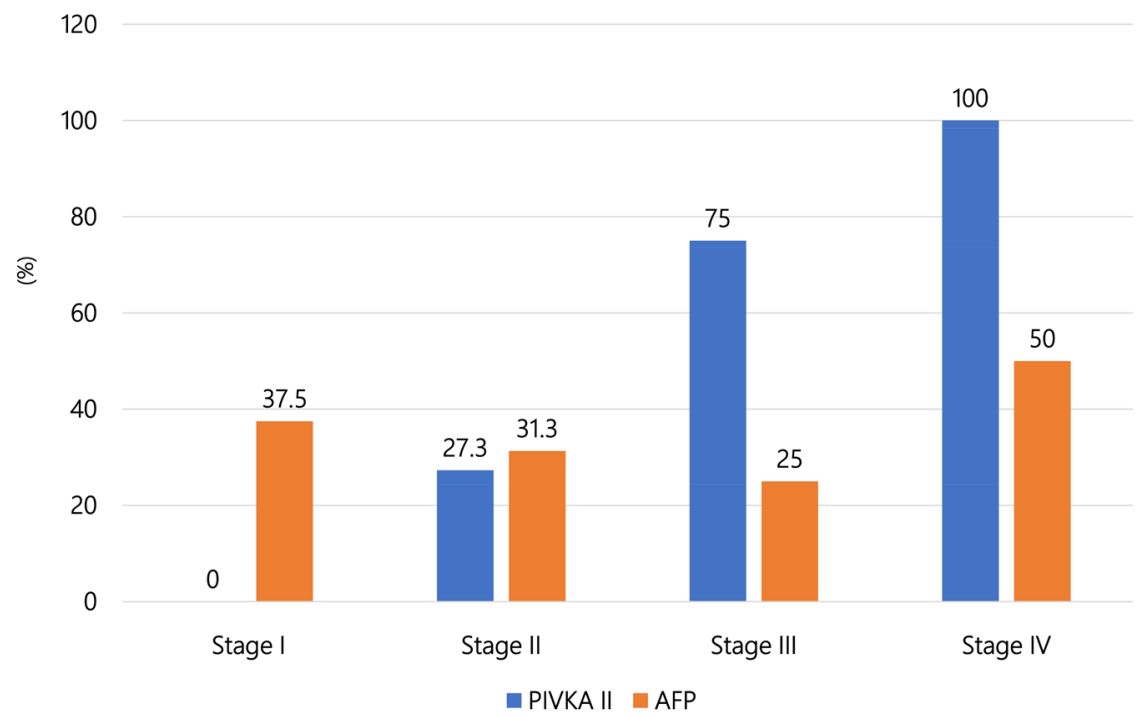

Figure 4. Positive rates (\%) of AFP and PIVKA-II at different stages of HCC. 
antigen-2 (DUPAN-2), and ferritin have also been used to diagnose pancreatic cancer. However, the combined use of CA19-9 and CA50 or Span-1 is recommended when CA19-9 is extremely low level. In the population, $5 \%-10 \%$ of individuals are Lewis antigen-negative, with no or low secretion of CA19-9 [21]. Lewis- negative pancreatic cancer is an aggressive subgroup with special clinical and molecular features [22]. There is an association of Lewis antigen phenotype $[\operatorname{Le}(a+b-), \operatorname{Le}(a-b+)$, and $\operatorname{Le}(a-b-)]$ with survival of patients with pancreatic ductal adenocarcinoma: the risk of mortality increases in the order of $\operatorname{Le}(a+b-)$, $\mathrm{Le}(\mathrm{a}-\mathrm{b}+)$, and $\mathrm{Le}(\mathrm{a}-\mathrm{b}-)$ [23]. Markers of sealyl Group A, including CA19-9, are elevated when pancreatic cancer, cholelithiasis, or Vater papillitis is complicated with obstructive jaundice. There are no specific tumor markers for biliary system cancers, but there are some cases where cancer cells produce sealyl Lewis antigen A, SLX, NCC-ST-439, or CEA.

- CA19-9:

CA19-9 attracted marked attention as a pancreatic cancer-specific marker because it was the first marker found to be effective for detecting pancreatic cancer. The positive rates of CA19-9 are $90 \%$ for pancreas cancer, $40 \% \sim 80 \%$ for biliary system cancer, and about $30 \%$ for gastric and colorectal cancer, suggesting that its utility is highest for digestive system cancers. The CA19-9 values in cases of pancreatic cancers tend to be 100,000 - 1,000,000 U/ml. Among benign diseases, the positive rate of CA19-9 is $20 \%$ for cholelithiasis; the value is approximately $\leq$ $10 \%$ for other benign disorders (Figure 5).

4) Lung cancer

Because tumor markers have low utility for the early diagnosis of lung cancer, their clinical utility is inferior to that of an imaging diagnosis. However, the tumor marker value reflects the stage and histologic type of lung cancer, suggesting

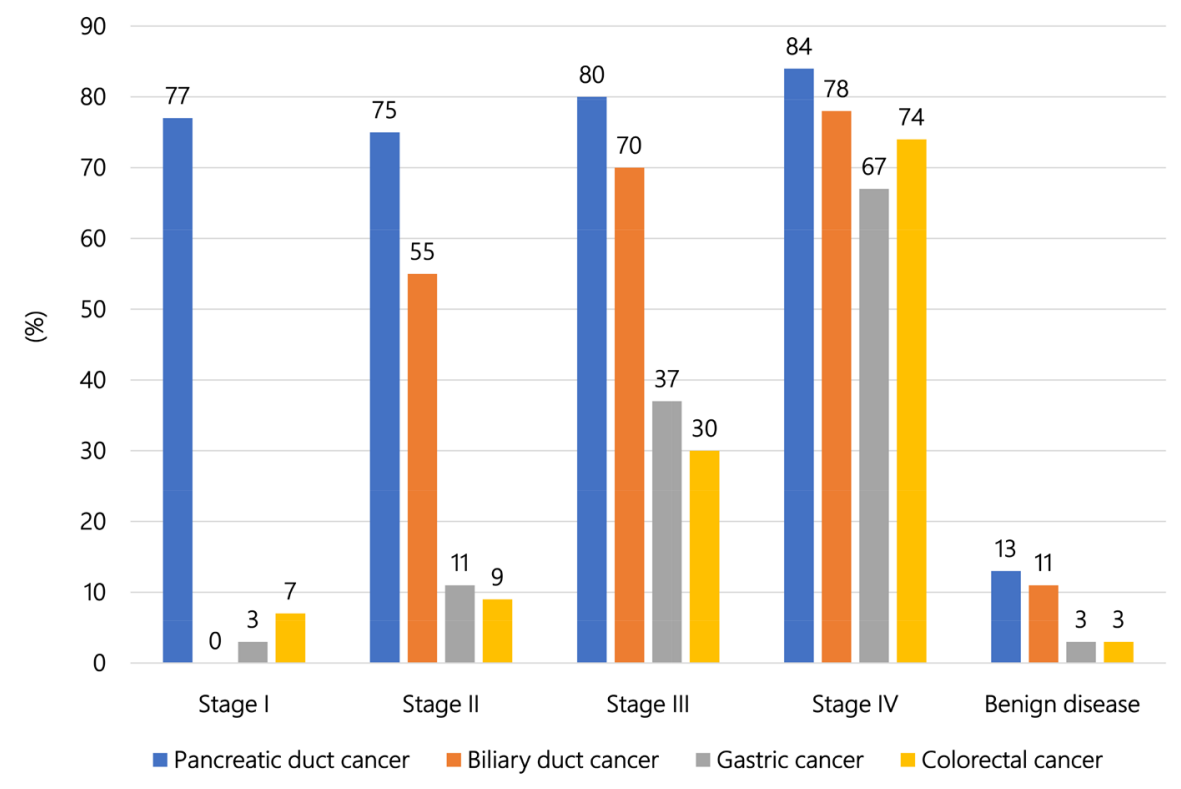

Figure 5. Positive rates (\%) of CA19-9 at different stages of pancreatic duct, biliary tract, gastric, and colorectal cancers. 
it to be essential for clinical diagnosis of lung cancer. In the clinical diagnosis of lung cancer, useful tumor markers include CEA, SLX, SCC antigen, cytokeratin 19 fragment (CYFRA 21-1), NSE, and progastrin-releasing peptide (ProGRP). Lung cancer has a variety of histological types: CEA has a high positive rate in all types of lung cancer, and high specificities of SLX, SCC antigen/CYFRA 21-1, and NSE/proGRP are found in lung adenocarcinoma, squamous cell carcinoma, and small cell carcinoma, respectively (Figure 6). Quantitative measurements of these serum tumor markers allow us to make supporting diagnoses of lung cancer, suggest the histologic type, help determine the clinical stage, aid in judging the effects of treatment, and monitoring follow-up. Nakamura and Nishimura [24] elegantly reviewed the molecular features, functions, and clinical relevance of conventional serum biomarkers for lung cancer, such as CEA, CYFRA 21-1, TPA, CA19-9, SLX, CA-125, SCC-antigen, NSE, and proGRP, and provided a snapshot of the current landscape and their potential combined utility in the diagnosis and prognosis of lung cancer.

5) Breast cancer

Serum tumor markers, such as CA 15-3 and breast cancer antigen 225 (BCA225), are combined use for diagnosing mammary cancer. However, these markers are not powerful for detecting early breast cancer [25] [26] [27]. CEA and NCC-ST-439 are other tumor markers that show a high positive rate for detecting breast cancer [25] [26] [27].

Non-palpable "T0 breast cancer" can be detected by measuring the CEA level in the milk secretion. HER2 protein produced by the proto-oncogene c-erb2 is found in high-grade breast cancer. It has a structure similar to that of epidermal growth factor receptor (EGFR). The measurement of HER-2 is important for determining the most appropriate treatment, as monoclonal antibody drugs

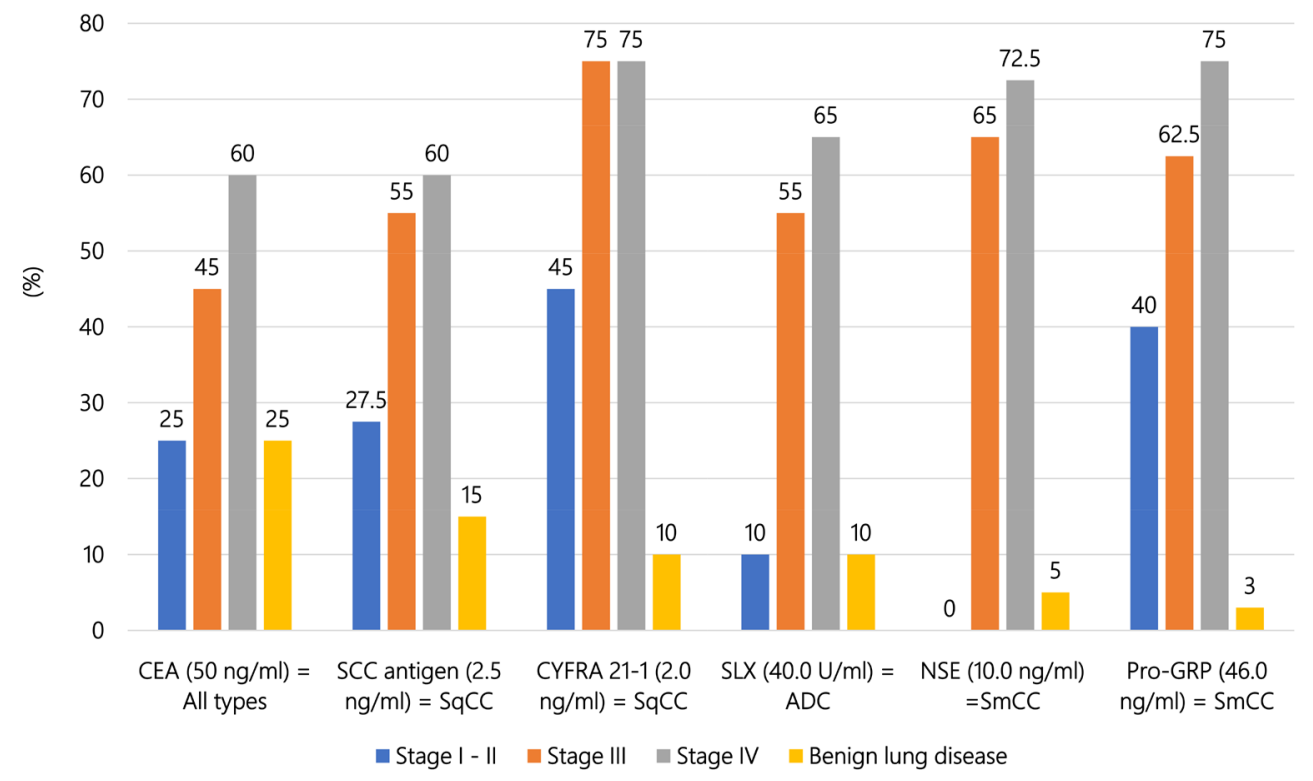

Figure 6. Various tumor markers for lung cancer. Reference values, association between stage and histological types, stages, positive rates, and false-positive rates. 
against this protein have recently been introduced. The positive rates of various tumor markers for breast cancer at different clinical stages are illustrated in Figure 7.

Data on the detection of non-palpable T0 breast cancer by measuring the CEA level in milk secretion are shown in Figure 8.

Regarding the measurement of serum or tissue HER2 protein levels, the positive rate of serum HER2 protein is 50\% in cases with the postoperative recurrence of HER2 over-expressing breast cancer. A previous report found that the rate was $80 \%-90 \%$ when measured in cases that were HER2 protein-positive on preoperative histopathology. Cases of HER2-overexpressing breast cancers, frequently show metastasis and recurrence, so the serum HER2 protein measurement is useful as a marker of postoperative recurrence of such cancer. In addition, HER2 measurement is important from a therapeutic aspect, as molecular-targeted drugs, such as trastuzumab (Herceptin), have recently been introduced and shown to be

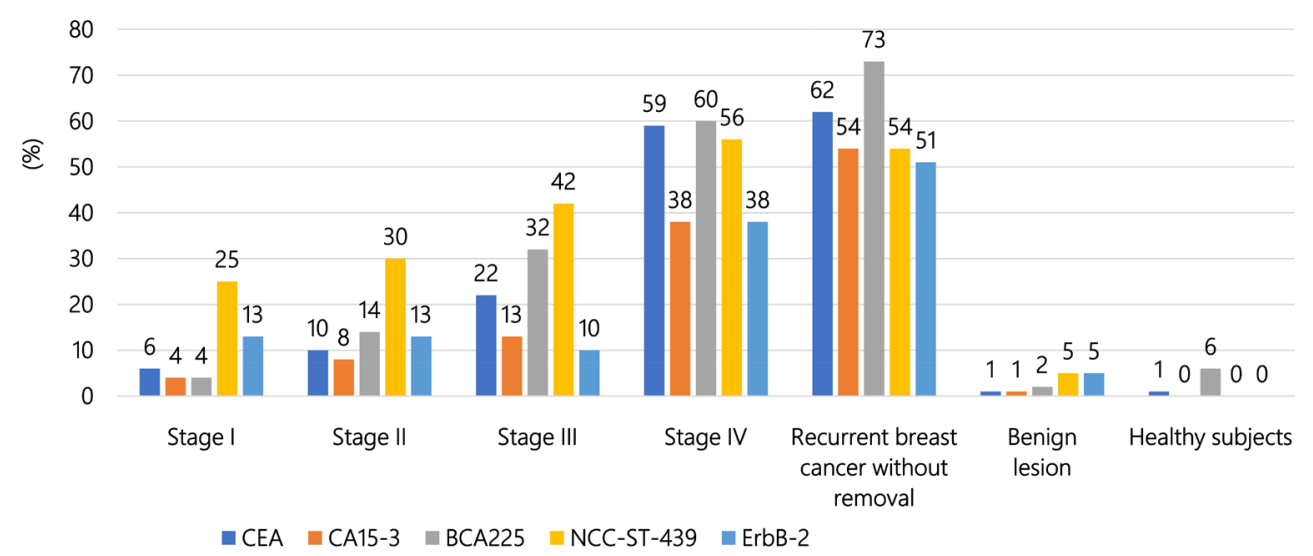

Figure 7. Positive rates (\%) of various tumor markers at different stages of breast cancer.

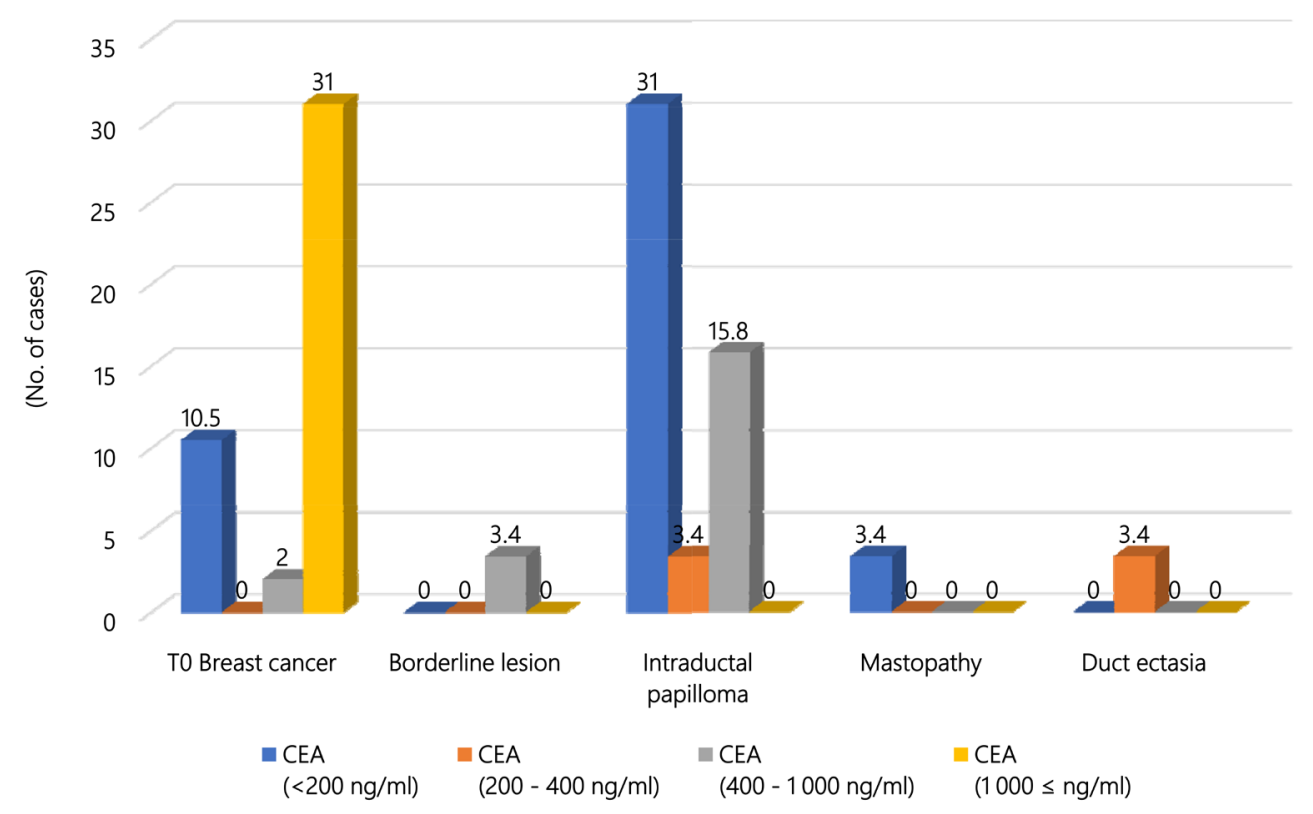

Figure 8. CEA values in nipple discharge from non-palpable mammary duct lesions. 
effective for HER2 protein-overexpressing breast cancer [28].

6) Gynecologic organ cancer

- Cervical cancer

The majority (90\%) of uterine cervical cancers are SCC, accounting for $90 \%$ of cases, followed by adenocarcinoma. We therefore first select SCC antigen and then CA125 for detecting cervical adenocarcinoma. CA125 is also useful for detecting uterine corpus carcinoma, fallopian tube cancer, and uterine leiomyoma, although there are no specific tumor markers for these tumors.

- Ovarian cancer

The frequency of ovarian malignancy is low, but its fatality rate is high. The ovary is located in the pelvic cavity, and the initial symptoms of ovarian malignancy are few. Therefore, ovarian cancer is called "a silent tumor" and is often discovered at an advanced stage, proving a major concern for gynecologists. Given this situation, the investigation of tumor markers can be quite useful.

In addition, ovarian cancer has a variety of histological types, so various types of tumor markers have been reported. Because the early detection of ovarian cancer is difficult, screening with combination assays has been attempted. We have used AFP [29] [30] [31] to detect germ cell tumors and CA125 [32] [33] [34] [35] to identify epithelial ovarian tumors. The positive rates of serum CA125 in different histologic types of ovarian cancer are shown in Figure 9, and the positive rates of various tumor markers at different stages of ovarian cancer are illustrated in Figure 10.

- Choriocarcinoma

Similar to other countries [36] [37] [38] [39], in Japan, the management of trophoblastic diseases, including choriocarcinoma by hCG, hCG $\beta$ or C-terminal peptide (hCG $\beta$-CTP) is prescribed in the handling agreement of the Japan Society

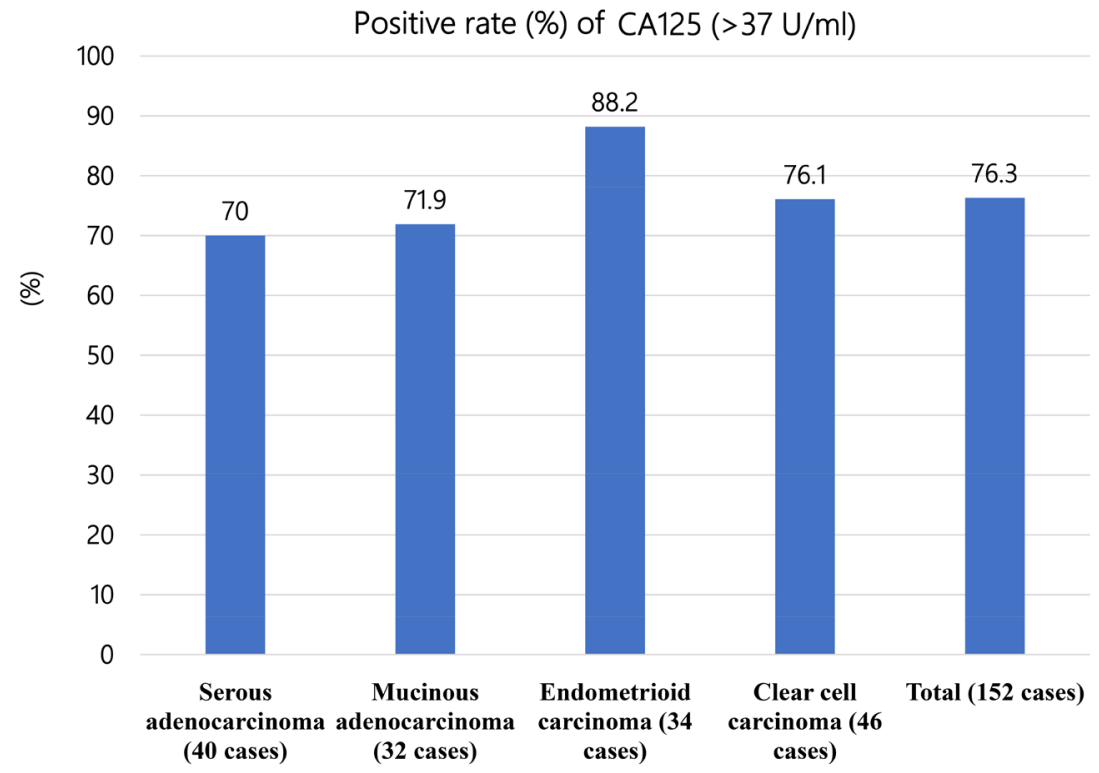

Figure 9. Positive rate (\%) of CA125 (>37 U/ml) according to the histopathological type of ovarian cancer. 


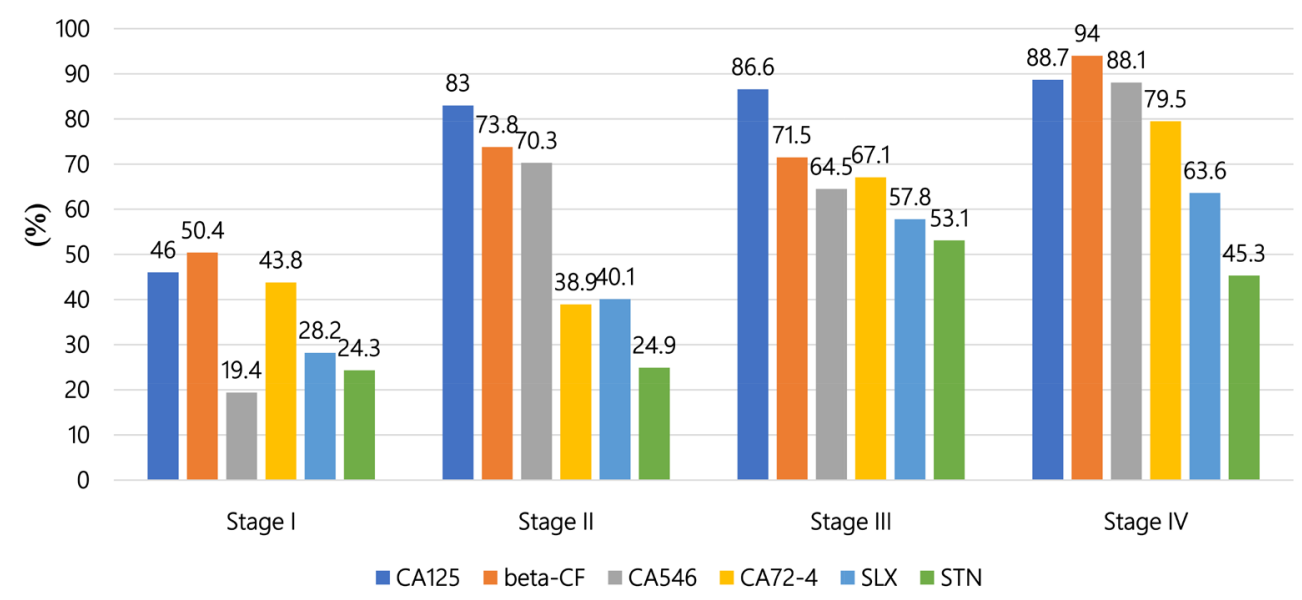

Figure 10. Positive rate (\%) of various tumor markers at different stages of ovarian cancer.

of Obstetrics and Gynecology [40] [41] [42].

7) Urogenital cancer

\section{- Prostate cancer}

PSA is a serine protease, and liquefaction of the sperm after ejaculation is widely recognized as its physiological function. Blood PSA forms a complex with $\alpha 1$-anti-chymotripsin (ACT), and this complex can be recognized with an anti-PSA antibody. However, a small portion of PSA binds to an $\alpha 2$ macroglobulin, becoming masked, and thus difficult to detect with the anti-PSA antibody. The utility of PSA is widely recognized, and it is frequently used for screening, making a diagnosis, determining the effect of treatment, and following patients with prostatic cancer [43] [44] [45] [46] [47].

However, it is difficult to differentiate prostatic cancer from prostatic hyperplasia when the value of PSA is low. Therefore, other markers, such as the free/total PSA ratio (\%PSA), PSA-ACT complex, and pro-PSA (pPSA), are used to differentiate prostate cancer, with good results obtained. The PSA level becomes lower by total prostatectomy or various treatments of prostate cancer. Therefore, highly sensitive PSA assays have been developed for the early detection of prostate cancer recurrence after treatment.

Differentiating prostatic hypertrophy and prostate cancer:

$\diamond$ Cut-off value for total PSA

In American cases, serum PSA levels of $<4 \mathrm{ng} / \mathrm{ml}, 4-10 \mathrm{ng} / \mathrm{ml}$, and $\geq 10 \mathrm{ng} / \mathrm{ml}$ are considered reference values, gray zone, and abnormal, respectively. Using 4 $\mathrm{ng} / \mathrm{ml}$ as the cut-off point, $43 \%$ of prostate cancer cases are false-negative, and $25 \%$ of prostatic hypertrophy are false-positive. As shown in Figure 11, the range of $4-10 \mathrm{ng} / \mathrm{ml}$ of PSA is considered the gray zone, as the likelihood of prostate cancer and noncancerous prostatic disease is high with these values.

$\diamond$ Ratio of Free-PSA/Total-PSA (\%PSA)

When examining components of PSA for prostate cancer and prostatic hyperplasia, the ratio of PSA-ACT is high in prostate cancer, and the ratio of free-PSA is high for prostatic hyperplasia. The diagnostic sensitivity and specificity for 


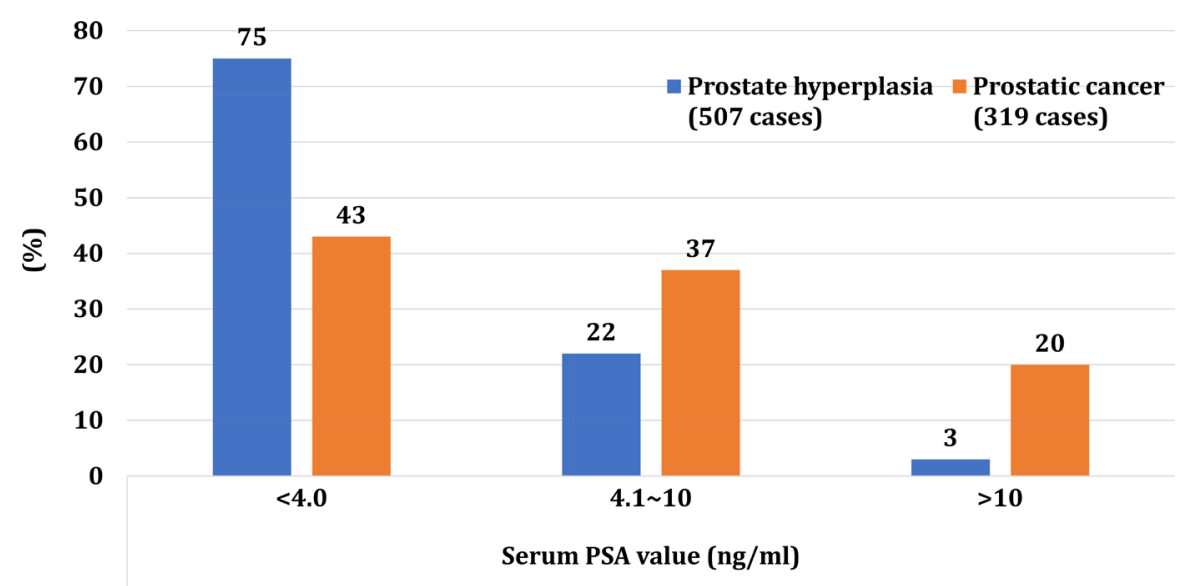

Figure 11. Percent distribution of serum PSA levels in patients with benign prostatic hyperplasia and prostatic cancer.

prostate cancer can be improved by measuring the \%PSA and measuring PSAACT and free-PSA at the same time. Zenimoto et al. [48] determined the \% PSA in 25 prostate cancer patients and 22 prostatic hypertrophy patients, finding that free-PSA ratio was low level for prostate cancer patients in the gray zone with a mildly increased total-PSA ratio. They set the cutoff value of \%PSA with approximately $20 \%$ or less for differentiation between prostate cancer and prostatic hypertrophy.

\section{$\diamond$ PSA-ACT complex}

The measurement of the PSA-ACT complex is not yet common, but it can be determined by a sandwich immunoassay using one antibody recognizing the PSA moiety and one recognizing the ACT part. According to a report comparing the diagnostic efficiency of prostate cancer with this complex and that of the total-PSA level, the efficiency with the complex was superior, possibly because factors other than the tumor, such as aging, may affect the free-PSA level.

$\diamond$ pPSA

Among the three molecular forms that constitute free-PSA (proPSA, BPSA, and inPSA), proPSA is an imperfect PSA precursor with incomplete cleavage of the N-terminal peptide and is produced abundantly by prostatic cancer cells. Therefore, its increase in the serum is expected to be a useful specific new marker of prostatic cancer [45].

- Testicular tumors

Measuring tumor markers is useful for detecting testicular germ cell tumors [49] [50] [51]. Testicular choriocarcinoma contains tumorous syncytial trophoblasts that produce hCG, so a high level of serum hCG is always detected in such case (Figure 12). Approximately $10 \%$ of seminoma cases may include syncytial trophoblasts and produce hCG (Figure 12). The two biomarkers, AFP and the hCG $\beta$ subunit, are also useful for detecting the presence of a residual tumor and determining the efficacy of treatment (Figure 12).

- Bladder cancer

It is important to identify the recurrence of bladder cancer early, as the 


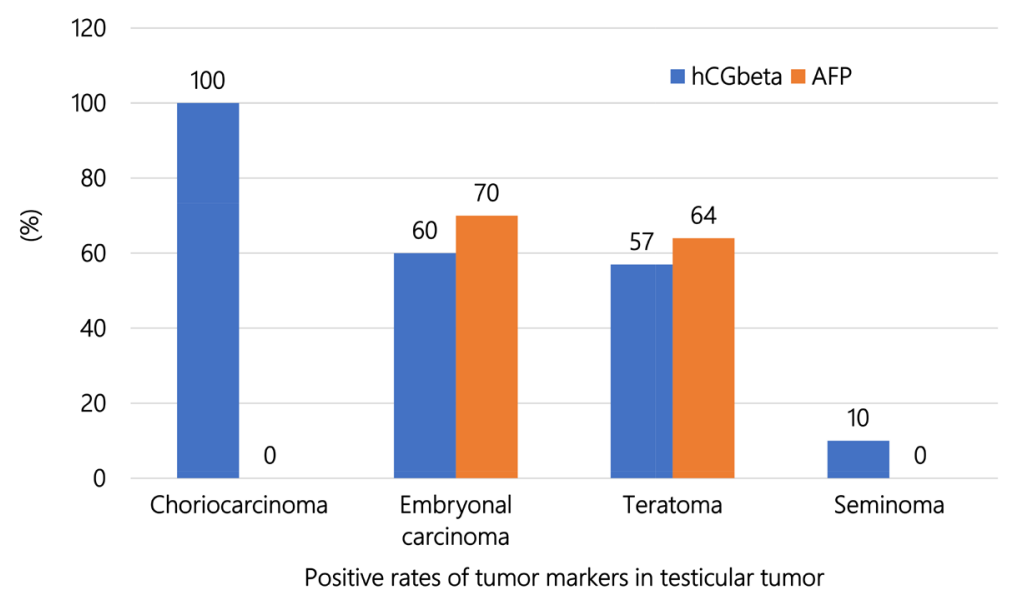

Figure 12. Positive rates (\%) of hCG $\beta$ and AFP in testicular tumors.

recurrence rate of urothelial cancer is high, and early detection can extend the patient's survival. In general, bladder tumor antigen (BTA) and nuclear matrix protein 22 (NMP22) are used for the detection of bladder cancer [52]-[57].

8) Pitfall of the abnormal value of tumor markers

- CEA: Its high level is naturally high in a large population of the smoker, liver cirrhosis patients, diabetics, and elderly people.

- AFP: The level is increased in cases of chronic hepatitis, liver cirrhosis, and pregnancy.

- TPA: False-positives are frequent, and levels are increased in cases of infection and pregnancy.

- SLX: Levels are increased in cases positive for lung cancer and Lewis antigen negative $[\mathrm{Le}(\mathrm{a}-\mathrm{b}-)]$.

- CA19-9: Levels are increased in gastrointestinal carcinomas, including pancreatic cancer, but negative in $\operatorname{Le}(\mathrm{a}-\mathrm{b}-)$.

- SCC: Levels are increased in cases of skin disease.

- CA125: Levels are increased in cases of endometriosis, menstruating patients, cases of peritonitis, pregnant patients, and liver cirrhosis patients.

\section{Conclusion}

Tumor markers comprise a wide spectrum of biomacromolecules excessively synthesized by a variety of neoplastic cells. These markers can be endogenous products of highly active metabolites from malignant neoplastic cells or the products of newly activated genes. Ideally, tumor markers should be highly sensitive, specific, and reliable with a high prognostic value and organ specificity. In addition, they should reflect the tumor stage. However, no tumor markers identified thus far have all of these characteristics. Nevertheless, most tumor markers show excellent clinical relevance for monitoring the efficacy of a variety of therapies.

\section{Conflicts of Interest}

The authors declare no conflicts of interest regarding the publication of this paper. 


\section{References}

[1] Sorensen, C.G., Karlsson, W.K., Pommergaard, H.C., Burcharth, J. and Rosenberg, J. (2016) The Diagnostic Accuracy of Carcinoembryonic Antigen to Detect Colorectal Cancer Recurrence-A Systematic Review. International Journal of Surgery, 25, 134-144. https://doi.org/10.1016/j.ijsu.2015.11.065

[2] Ohyanagi, H., Saitoh, Y., Okumura, S., Ishida, T., Uesaka, K., Ishida, H., Yamamoto, M., Uemura, Y., Kano, Y. and Suyama, T. (1987) A New Monoclonal-Antibody-Defined Tumor Marker (KMO1) for Pancreatic Carcinoma. Mount Sinai Journal of Medicine, 54, 393-400.

[3] Gold, P. and Freedman, S.O. (1965) Demonstration of Tumor-Specific Antigens in Human Colonic Carcinomata by Immunological Tolerance and Absorption Techniques. Journal of Experimental Medicine, 121, 439-462. https://doi.org/10.1084/jem.121.3.439

[4] Jelski, W. and Mroczko, B. (2019) Biochemical Diagnostics of Pancreatic Cancer-Present and Future. Clinica Chimica Acta, 498, 47-51.

https://doi.org/10.1016/j.cca.2019.08.013

[5] Shinozaki, E., Tanabe, K., Akiyoshi, T., Tsuchida, T., Miyazaki, Y., Kojima, N., Igarashi, M., Ueno, M., Suenaga, M., Mizunuma, N., Yamaguchi, K., Nakayama, K., Iijima, S. and Yamaguchi, T. (2018) Serum Leucine-Rich Alpha-2-Glycoprotein-1 with Fucosylated Triantennary N-Glycan: A Novel Colorectal Cancer Marker. BMC Cancer, 18, 406. https://doi.org/10.1186/s12885-018-4252-6

[6] Si, Y.Q., Wang, X. Q., Fan, G., Wang, C.Y., Zheng, Y.W., Song, X., Pan, C.C., Chu, F.L., Liu, Z.F., Lu, B.R. and Lu, Z.M. (2020) Value of AFP and PIVKA-II in Diagnosis of HBV-Related Hepatocellular Carcinoma and Prediction of Vascular Invasion and Tumor Differentiation. Infectious Agents and Cancer, 15, 70.

[7] Tarao, K., Nozaki, A., Komatsu, H., Komatsu, T., Taguri, M., Tanaka, K., Chuma, M., Numata, K. and Maeda, S. (2020) Real Impact of Tumor Marker AFP and PIVKA-II in Detecting Very Small Hepatocellular Carcinoma $(</=2 \mathrm{~cm}$, Barcelona Stage 0)-Assessment with Large Number of Cases. World Journal of Hepatology, 12, 1046-1054. https://doi.org/10.4254/wjh.v12.i11.1046

[8] Saitta, C., Raffa, G., Alibrandi, A., Brancatelli, S., Lombardo, D., Tripodi, G., Raimondo, G. and Pollicino, T. (2017) PIVKA-II Is a Useful Tool for Diagnostic Characterization of Ultrasound-Detected Liver Nodules in Cirrhotic Patients. Medicine (Baltimore), 96, e7266. https://doi.org/10.1097/MD.0000000000007266

[9] Wang, B.L., Tan, Q.W., Gao, X.H., Wu, J. and Guo, W. (2014) Elevated PIVKA-II Is Associated with Early Recurrence and Poor Prognosis in BCLC 0-A Hepatocellular Carcinomas. Asian Pacific Journal of Cancer Prevention, 15, 6673-6678. https://doi.org/10.7314/APJCP.2014.15.16.6673

[10] Wu, J., Xiang, Z., Bai, L., He, L., Tan, L., Hu, M. and Ren, Y. (2018) Diagnostic Value of Serum PIVKA-II Levels for BCLC Early Hepatocellular Carcinoma and Correlation with HBV DNA. Cancer Biomark, 23, 235-242. https://doi.org/10.3233/CBM-181402

[11] Huang, S., Jiang, F., Wang, Y., Yu, Y., Ren, S., Wang, X., Yin, P. and Lou, J. (2017) Diagnostic Performance of Tumor Markers AFP and PIVKA-II in Chinese Hepatocellular Carcinoma Patients. Tumor Biology, 39, 1010428317705763. https://doi.org/10.1177/1010428317705763

[12] Han, K. and Kim, J.H. (2015) Transarterial Chemoembolization in Hepatocellular Carcinoma Treatment: Barcelona Clinic Liver Cancer Staging System. World Journal of Gastroenterology, 21, 10327-10335. https://doi.org/10.3748/wjg.v21.i36.10327 
[13] Kawano, Y., Sasaki, A., Kai, S., Endo, Y., Iwaki, K., Uchida, H., Shibata, K., Ohta, M. and Kitano, S. (2008) Short- and Long-Term Outcomes after Hepatic Resection for Hepatocellular Carcinoma with Concomitant Esophageal Varices in Patients with Cirrhosis. Annals of Surgical Oncology, 15, 1670-1676. https://doi.org/10.1245/s10434-008-9880-7

[14] Afshar, M., Fletcher, P., Bardoli, A.D., Ma, Y.T. and Punia, P. (2018) Non-Secretion of AFP and Neutrophil Lymphocyte Ratio as Predictors for Survival in Hepatocellular Carcinoma Patients Treated with Sorafenib: A Large UK Cohort. Oncotarget, 9, 16988-16995. https://doi.org/10.18632/oncotarget.24769

[15] Hiraoka, A., Ishimaru, Y., Kawasaki, H., Aibiki, T., Okudaira, T., Toshimori, A., Kawamura, T., Yamago, H., Nakahara, H., Suga, Y., Azemoto, N., Miyata, H., Miyamoto, Y., Ninomiya, T., Hirooka, M., Abe, M., Matsuura, B., Hiasa, Y. and Michitaka, K. (2015) Tumor Markers AFP, AFP-L3, and DCP in Hepatocellular Carcinoma Refractory to Transcatheter Arterial Chemoembolization. Oncology, 89, 167-174. https://doi.org/10.1159/000381808

[16] Dauti, F., Hjaltalin Jonsson, M., Hillarp, A., Bentzer, P. and Schott, U. (2015) Perioperative Changes in PIVKA-II. Scandinavian Journal of Clinical and Laboratory Investigation, 75, 562-567. https://doi.org/10.3109/00365513.2015.1058521

[17] Eguchi, S., Takatsuki, M., Hidaka, M., Soyama, A., Tomonaga, T., Muraoka, I. and Kanematsu, T. (2010) Predictor for Histological Microvascular Invasion of Hepatocellular Carcinoma: A Lesson from 229 Consecutive Cases of Curative Liver Resection. World Journal of Surgery, 34, 1034-1038.

https://doi.org/10.1007/s00268-010-0424-5

[18] Fujiki, M., Takada, Y., Ogura, Y., Oike, F., Kaido, T., Teramukai, S. and Uemoto, S. (2009) Significance of Des-Gamma-Carboxy Prothrombin in Selection Criteria for Living Donor Liver Transplantation for Hepatocellular Carcinoma. American Journal of Transplantation, 9, 2362-2371. https://doi.org/10.1111/j.1600-6143.2009.02783.x

[19] Shirabe, K., Itoh, S., Yoshizumi, T., Soejima, Y., Taketomi, A., Aishima, S. and Maehara, Y. (2007) The Predictors of Microvascular Invasion in Candidates for Liver Transplantation with Hepatocellular Carcinoma- with Special Reference to the Serum Levels of Des-Gamma-Carboxy Prothrombin. Journal of Surgical Oncology, 95, 235-240. https://doi.org/10.1002/jso.20655

[20] Li, D., Mallory, T. and Satomura, S. (2001) AFP-L3: A New Generation of Tumor Marker for Hepatocellular Carcinoma. Clinica Chimica Acta, 313, 15-19.

https://doi.org/10.1016/S0009-8981(01)00644-1

[21] Guo, M., Luo, G., Lu, R., Shi, W., Cheng, H., Lu, Y., Jin, K., Yang, C., Wang, Z., Long, J., Xu, J., Ni, Q., Liu, C. and Yu, X. (2017) Distribution of Lewis and Secretor Polymorphisms and Corresponding CA19-9 Antigen Expression in a Chinese Population. FEBS Open Bio, 7, 1660-1671. https://doi.org/10.1002/2211-5463.12278

[22] Liu, C., Deng, S., Jin, K., Gong, Y., Cheng, H., Fan, Z., Qian, Y., Huang, Q., Ni, Q., Luo, G. and Yu, X. (2020) Lewis Antigennegative Pancreatic Cancer: An Aggressive Subgroup. International Journal of Oncology, 56, 900-908. https://doi.org/10.3892/ijo.2020.4989

[23] Kwon, S., Kim, S., Giovannucci, E.L., Hidalgo, M., Markey, M.K., Bovik, A.C., Kwon, M.J., Kim, K.J., Im, H., Park, J.Y., Bang, S., Park, S.W., Song, S.Y. and Chung, M.J. (2020) Lewis Antigen Phenotype and Survival of Patients with Pancreatic Cancer. Pancreas, 49, 1348-1354.

https://doi.org/10.1097/MPA.0000000000001687 
[24] Nakamura, H. and Nishimura, T. (2017) History, Molecular Features, and Clinical Importance of Conventional Serum Biomarkers in Lung Cancer. Surgery Today, 47, 1037-1059. https://doi.org/10.1007/s00595-017-1477-y

[25] Ibusuki, M., Fujimori, H., Yamamoto, Y., Ota, K., Ueda, M., Shinriki, S., Taketomi, M., Sakuma, S., Shinohara, M., Iwase, H. and Ando, Y. (2009) Midkine in Plasma as a Novel Breast Cancer Marker. Cancer Science, 100, 1735-1739. https://doi.org/10.1111/j.1349-7006.2009.01233.x

[26] Iwase, H., Kobayashi, S., Itoh, Y., Fukuoka, H., Kuzushima, T., Iwata, H., Yamashita, T., Naitoh, A., Itoh, K. and Masaoka, A. (1995) Evaluation of Serum Tumor Markers in Patients with Advanced or Recurrent Breast Cancer. Breast Cancer Research and Treatment, 33, 83-88. https://doi.org/10.1007/BF00666074

[27] Ri, G., Ohno, S., Yamamoto, T., Ito, E., Furutani, M., Furutani, Y., Umeda, Y., Tsukahara, T., Hagita, N. and Matsuoka, R. (2009) Serum Levels of CA15-3, KL-6 and BCA225 Are Positively Correlated with Each Other in the General Population. Anticancer Research, 29, 4239-4242.

[28] Wilson, F.R., Coombes, M.E., Wylie, Q., Yurchenko, M., Brezden-Masley, C., Hutton, B., Skidmore, B. and Cameron, C. (2017) Herceptin(R) (Trastuzumab) in HER2-Positive Early Breast Cancer: Protocol for a Systematic Review and Cumulative Network Meta-Analysis. Systematic Reviews, 6, 196. https://doi.org/10.1186/s13643-017-0588-2

[29] Aziz, M.F. (1995) Current Management of Malignant Germ Cell Tumor of the Ovary. Gan To Kagaku Ryoho, 22, 262-276.

[30] Mann, J.R., Pearson, D., Barrett, A., Raafat, F., Barnes, J.M. and Wallendszus, K.R. (1989) Results of the United Kingdom Children's Cancer Study Group's Malignant Germ Cell Tumor Studies. Cancer, 63, 1657-1667.

https://doi.org/10.1002/1097-0142(19900501)63:9<1657::AID-CNCR2820630902>3. $\underline{0 . \mathrm{CO} ; 2-8}$

[31] Sengar, A.R. and Kulkarni, J.N. (2010) Growing Teratoma Syndrome in a Post Laparoscopic Excision of Ovarian Immature Teratoma. Journal of Gynecologic Oncology, 21, 129-131. https://doi.org/10.3802/jgo.2010.21.2.129

[32] Gandhi, T. and Bhatt, H. (2021) Cancer Antigen 125. StatPearls, Treasure Island.

[33] Briese, V. and Strache, R.R. (1988) Tumor Markers in Gynecology. General Review. Zentralblatt für Gynäkologie, 110, 257-266.

[34] Muinao, T., Deka Boruah, H.P. and Pal, M. (2019) Multi-Biomarker Panel Signature as the Key to Diagnosis of Ovarian Cancer. Heliyon, 5, e02826.

https://doi.org/10.1016/j.heliyon.2019.e02826

[35] Ugrinska, A., Bombardieri, E., Stokkel, M.P., Crippa, F. and Pauwels, E.K. (2002) Circulating Tumor Markers and Nuclear Medicine Imaging Modalities: Breast, Prostate and Ovarian Cancer. The Quarterly Journal of Nuclear Medicine, 46, 88104.

[36] Braga, A., Elias, K.M., Horowitz, N.S. and Berkowitz, R.S. (2021) Treatment of High-Risk Gestational Trophoblastic Neoplasia and Chemoresistance/Relapsed Disease. Best Practice \& Research: Clinical Obstetrics \& Gynaecology. https://doi.org/10.1016/j.bpobgyn.2021.01.005

[37] Bruce, S. and Sorosky, J. (2021) Gestational Trophoblastic Disease. StatPearls, Treasure Island.

[38] Fehlmann, A., Benkortbi, K., Rosseel, G., Meyer-Hamme, U., Tille, J.C., Sloan-Bena, F., Paoloni-Giacobino, A., Rougemont, A.L., Bodmer, A., Botsikas, D., Mathevet, P., 
Petignat, P. and Undurraga Malinverno, M. (2021) Gestational Trophoblastic Disease in Switzerland: Retrospective Study of the Impact of a Regional Reference Centre. Swiss Medical Weekly, 151, w20406. https://doi.org/10.4414/smw.2021.20406

[39] Mylvaganam, G., Allanson, E., Allanson, B., Philp, S., Pather, S., Farrell, R., Carter, J. and Tejada-Berges, T. (2020) Assessment of Current Follow-Up for Complete Molar Pregnancies: A Single Centre Review. The Australian and New Zealand Journal of Obstetrics and Gynaecology, 61, 213-216. https://doi.org/10.1111/ajo.13258

[40] Aoki, D., Hirasawa, A. and Susumu, N. (2005) Diagnostic Significance of Tumor Markers for Gynecologic Malignancies. Gan To Kagaku Ryoho, 32, 411-416.

[41] Sasaki, S. (2003) Management of Gestational Trophoblastic Diseases in Japan-A Review. Placenta, 24, S28-S32. https://doi.org/10.1053/plac.2002.0933

[42] Seki, K., Matsui, H. and Sekiya, S. (2004) Advances in the Clinical Laboratory Detection of Gestational Trophoblastic Disease. Clinica Chimica Acta, 349, 1-13. https://doi.org/10.1016/j.cccn.2004.04.027

[43] Becker, C. and Lilja, H. (1997) Individual Prostate-Specific Antigen (PSA) Forms as Prostate Tumor Markers. Clinica Chimica Acta, 257, 117-132. https://doi.org/10.1016/S0009-8981(96)06437-6

[44] Lilja, H. (1993) Significance of Different Molecular Forms of Serum PSA. The Free, Noncomplexed Form of PSA versus That Complexed to Alpha 1-Antichymotrypsin. Urologic Clinics of North America, 20, 681-686. https://doi.org/10.1016/S0094-0143(21)00920-4

[45] Mikolajczyk, S.D. and Rittenhouse, H.G. (2004) Tumor-Associated Forms of Prostate Specific Antigen Improve the Discrimination of Prostate Cancer from Benign Disease. Rinsho Byori, 52, 223-230.

[46] Okihara, K. and Babaian, R.J. (2001) Complexed Prostate-Specific Antigen Improvement in Detecting Prostate Cancer. Current Urology Reports, 2, 253-258. https://doi.org/10.1007/s11934-001-0088-7

[47] Stenman, U.H., Leinonen, J., Zhang, W.M. and Finne, P. (1999) Prostate-Specific Antigen. Seminars in Cancer Biology, 9, 83-93.

https://doi.org/10.1006/scbi.1998.0086

[48] Zenimoto, M., Kita, M., Arai, T., Murayama, T. and Mori, M. (2003) Free/Total Prostate Specific Antigen Ratio (\%PSA) to Predict Probability of Prostate Cancer. Rinsho Byori, 51, 969-973.

[49] Bassetto, M.A., Franceschi, T., Lenotti, M., Parise, G., Pancheri, F., Sabbioni, R., Zaninelli, M. and Cetto, G.L. (1994) AFP and HCG in Germ Cell Tumors. The International Journal of Biological Markers, 9, 29-32. https://doi.org/10.1177/172460089400900106

[50] Ruther, U., Rothe, B., Grunert, K., Bader, H., Sessler, R., Nunnensiek, C., Rassweiler, J., Luthgens, M., Eisenberger, F. and Jipp, P. (1994) Role of Human Chorionic Gonadotropin in Patients with Pure Seminoma. European Urology, 26, 129-133. https://doi.org/10.1159/000475361

[51] Schwarz, H.P., Susani, M., Kratzik, C., Studler, G., Krisch, K. and Aiginger, P. (1984) Importance of the Intracellular Detection of Alpha-Fetoprotein and Beta-Human Chorionic Gonadotropin in a Testicular Tumor Using Immunoperoxidase. Wiener klinische Wochenschrift, 96, 230-234.

[52] Abd El Gawad, I.A., Moussa, H.S., Nasr, M.I., El Gemae, E.H., Masooud, A.M., Ibrahim, I.K. and El Hifnawy, N.M. (2005) Comparative Study of NMP-22, Telo- 
merase, and BTA in the Detection of Bladder Cancer. Journal of the Egyptian National Cancer Institute, 17, 193-202.

[53] Bhuiyan, J., Akhter, J. and O’Kane, D.J. (2003) Performance Characteristics of Multiple Urinary Tumor Markers and Sample Collection Techniques in the Detection of Transitional Cell Carcinoma of the Bladder. Clinica Chimica Acta, 331, 69-77. https://doi.org/10.1016/S0009-8981(03)00074-3

[54] Chou, R., Gore, J.L., Buckley, D., Fu, R., Gustafson, K., Griffin, J.C., Grusing, S. and Selph, S. (2015) Urinary Biomarkers for Diagnosis of Bladder Cancer: A Systematic Review and Meta-Analysis. Annals of Internal Medicine, 163, 922-931. https://doi.org/10.7326/M15-0997

[55] Dey, P. (2004) Urinary Markers of Bladder Carcinoma. Clinica Chimica Acta, 340, 57-65. https://doi.org/10.1016/j.cccn.2003.11.008

[56] Jiang, Q., Han, T., Ren, H., Aziz, A.U.R., Li, N., Zhang, H., Zhang, Z. and Liu, B. (2020) Bladder Cancer Hunting: A Microfluidic Paper-Based Analytical Device. Electrophoresis, 41, 1509-1516. https://doi.org/10.1002/elps.202000080

[57] Kikuchi, H. (2004) Tumor Markers of Urinary Tract Carcinoma. Rinsho Byori, 52, 371-380. 


\section{Abbreviations:}

ACT, $\alpha 1$-anti-chymotripsin

AFP, $\alpha$-fetoprotein

AFP-L3, AFP lectin-responsive fractionation

BCA225, breast cancer antigen 225

$\mathrm{BPH}$, benign prostatic hyperplasia

BTA, bladder tumor antigen

I-CTP, carboxy-terminal telopeptide of type I collagen

$\mathrm{CA}$, carbohydrate antigen

CEA, carcinoembryonic antigen

CSLEX, Anti-sialyl-Lex(x) mAb

CYFRA 21-1, cytokeratin 19 fragment

Dupan-2, pancreatic cancer-associated antigen-2

hCG, human chorionic gonadotropin

hCG $\beta$, hCG beta-subunit

hCG $\beta$-CTP, hCG beta-carboxyl terminal peptide

HCC, hepatocellular carcinoma

HER2, human epidermal growth factor receptor type 2

KL-6, Krebs von den Lungen-6

LCA, Lens culinaris agglutinin

MUC-1, mucin-1

NCC-ST-439, Nation Cancer Center-Stomach-439

NMP22, nuclear matrix protein 22

NSE, neuron specific enolase

PIVKA-II, protein induced by vitamin $\mathrm{K}$ absence or antagonist-II

pPSA, pro prostate specific antigen

$\mathrm{PPV}$, positive predictive value

ProGRP, progastrin-releasing peptide

PSA, prostate-specific antigen

PTH, parathyroid hormone

PTHrP, parathyroid hormone-related peptide

SCC antigen, squamous cell carcinoma antigen

SLX, sialyl Le(x) antigen

SPAN-1, s-pancreas-1 antigen

STN, Sialyl Thomsen-nouveau

TPA, tissue polypeptide antigen 